\title{
Design of Smart Home System based on ARM and ZigBee Technology
}

\author{
Feng Tian, Shimin Wang and Yongtao Yu \\ College of Automation, Shenyang Aerospace University, Shenyang, 110136, China \\ shimin09@126.com
}

\begin{abstract}
This paper designs a portable smart home system which based on ARM embedded gateway and Zigbee wireless sensor network. With the low power consumption, ad-hoc network, scalability and high practicability of ZigBee technology, wireless sensor network is built in the home. The smart home system use S5PV210 processor as the core of Hardware platform and use embedded Linux operating system as real-time operating system, porting Web server for embedded gateway and develop a human-computer interaction interface with Qt, both local and remote can monitor and control the home appliance equipment. The feasibility of the design is verified by experiments. At the same time, the system is extensible and portable, so it will have a good reference value for the similar system.
\end{abstract}

Keywords: ARM embedded gateway, Linux operating system, Qt, Web server, ZigBee

\section{Introduction}

With the improvement of people's living standards and the development of computer technology, communication technology and network technology, smart home gradually become the future development of home life direction. The smart home system is a technology which integrates computer, communication and control technology. It can not only provides users with safe, healthy and comfortable living environment, but also can remote monitor their home state and control home appliance equipment. At present, there are more and more kinds of smart home products, but the low performance CPU is generally used as the master chip, so it can't integrate the more complex tasks in the smart home system and can't detect the work of electrical equipments. In this paper, a convenient, fast and efficient smart home system is designed based on the advanced CPU chip and ZigBee wireless sensor network technology.

\section{Function and Composition of System}

The overall structure of the smart home system is shown in Figure 1.

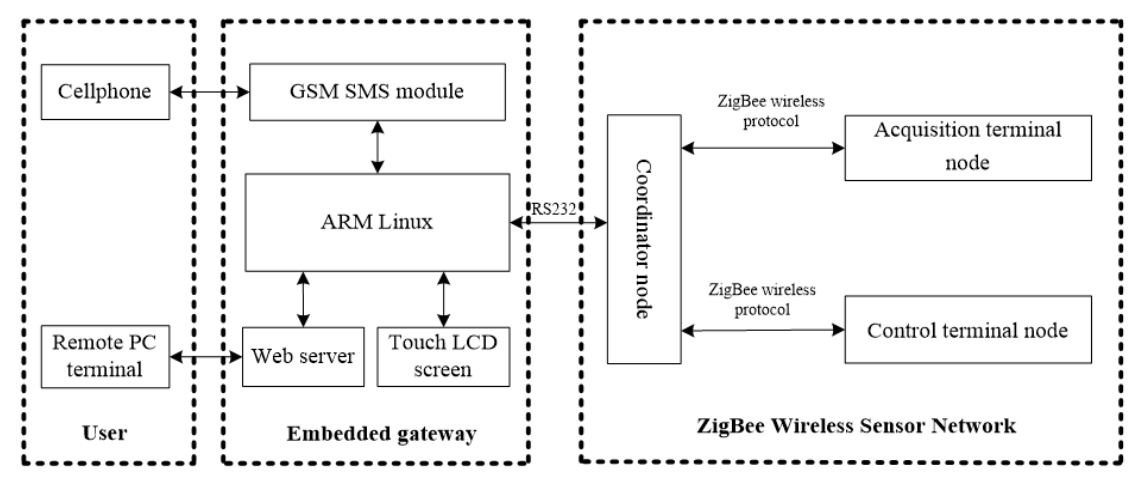

Figure 1. The Structure of the System 
The main components of the smart home system are embedded gateway, ZigBee coordinator node, ZigBee terminal node, GSM module, etc.

The embedded gateway is composed of embedded Web server, ZigBee coordinator and SMS transceiver GSM module. ZigBee coordinator dominates the home wireless network; the embedded Web server is responsible for communication with external network, it allows users to $\log$ to the Web server by a remote PC browser or a mobile browser.

The data of the transmission between the coordinator and the acquisition terminal node mainly include temperature and humidity, smog, gas, flame, water heater temperature, etc.

The data of the transmission between the coordinator and the acquisition terminal node mainly include control command and working status of electrical equipment.

The main functions of the smart home control system include:

(1) Control at local touch screen: The user can view the electrical status and control home appliance by the touch screen interface in the indoor.

(2) Indoor environment monitoring: The system can detect the temperature, humidity, gas and smoke of the home environment by the sensor.

(3) Automatic alarm: When detected the temperature, humidity, gas, smoke etc exceed the standard, the system will send an alert message to the user's mobile phone and automatically turn on the ventilator.

(4) Remote control by Web: Users can log in to the Web server of the embedded gateway by a remote PC browser or a mobile browser, remote control for home appliances and equipment at anytime and anywhere.

(5) Burglar alarm: When the user goes out turn on the alarm system, if the pyroelectric infrared sensors detects someone intrusion into the room, the system will send an alert message to the user.

(6) Detection of the electrical equipment malfunction: When the user opens the home appliance equipment, the system can detect the working status of the equipment, if the device does not work will alert the user.

\section{Hardware Design of System}

\subsection{Hardware Design of Terminal Node}

Because the system is only short distance information collection and control, no communication is needed between the terminal nodes; they only need to communicate with the coordinator node, so ZigBee network topology selection the star network. In order to reduce the cost, the system terminal node selects the half functional equipment (RFD) and the coordinator selects the full functional equipment (FFD). Choose CC2530 processor as ZigBee chip with designed by TI corporation, It includes RF transceiver, enhanced 8051MCU, programmable flash, It provides improved RF performance, robust address recognition and data packet processing engine over previous generation CC2430. CC2530 has different operating mode, which makes it especially adapted to the system of ultra-low power consumption.

The basic hardware block diagram of the ZigBee node is shown in Figure 2. 


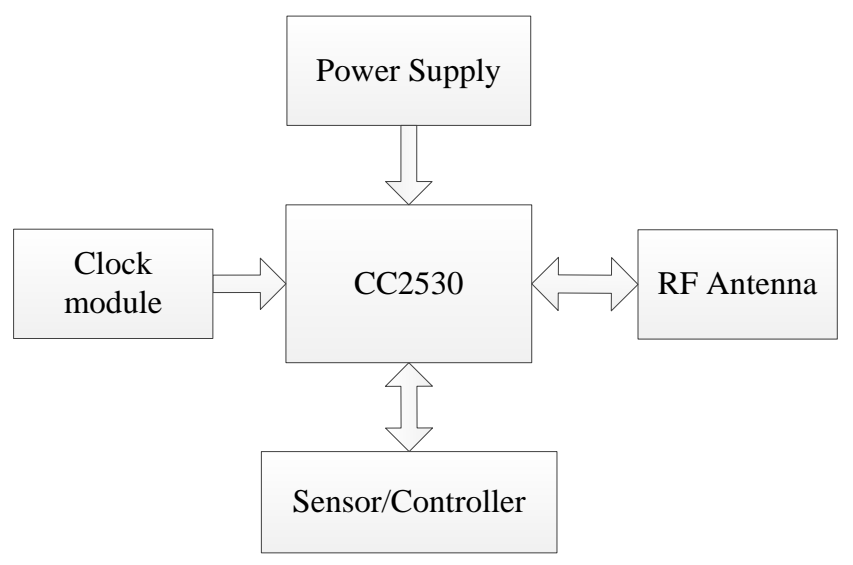

Figure 2. Hardware Block Diagram of ZigBee Node

The node of the control terminal can not only control the home appliance equipment, but also can detect the fault of the electrical equipment. When it controls the relay is closed, it will detect the current in the device. Control circuit and fault detection circuit schematic diagram shown in Figure 3. If the device works, the current transformer T1 output is relatively small AC current, the AC current signal is converted into AC voltage signal, and then the AC voltage signal is amplified and double voltage detection circuit output voltage signal to the CC2530 chip. If the CC2530 does not detect a voltage signal then the device does not work, so as to judge the fault of the equipment. After the experimental verification, the circuit can detect the normal operation of household appliances.

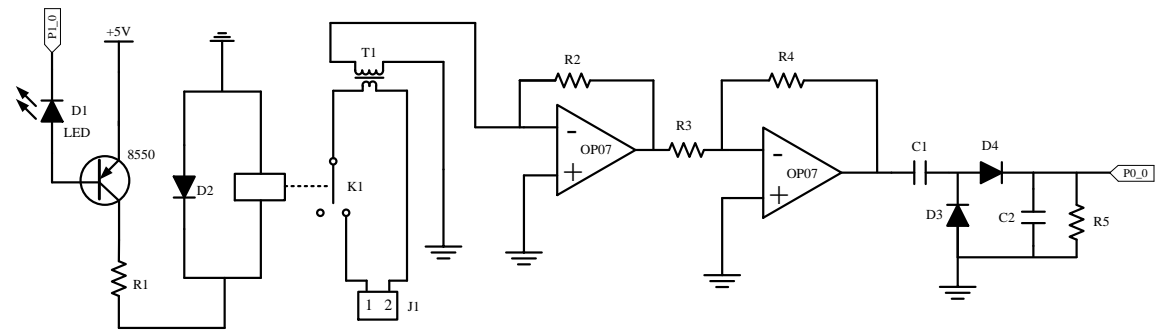

Figure 3. Schematic Diagram of Fault Detection Circuit

\subsection{Hardware Design of Embedded Gateway}

The gateway of smart home system is a network protocol converter which based on transport layer. It connects two or more than two separate networks to realize the conversion between the wireless sensor network and the Internet network protocol. The gateway not only can interconnection wide area network or LAN, but also have address management functions for the sensor nodes. Remote control function is realized by sending command of node address. The embedded gateway uses the S5PV210 processor as the core which in the Cortex-A8 architecture. The hardware platform is consisted of the external expansion memory, LCD touch screen, Internet interface, RS232 interface, etc.

The S5PV210 processor which is launched by Samsung Electronics Corporation is an application for smart phones and tablet PCs and other multimedia equipment applications processor. It uses the Cortex-A8 ARM kernel and its main frequency can reach 1GHZ, the internal bus structure with 64/32 bit. It can achieve the high performance of 2000DMIPS (200 millions instruction per second) computing.

The hardware structure diagram is shown in Figure 4. 


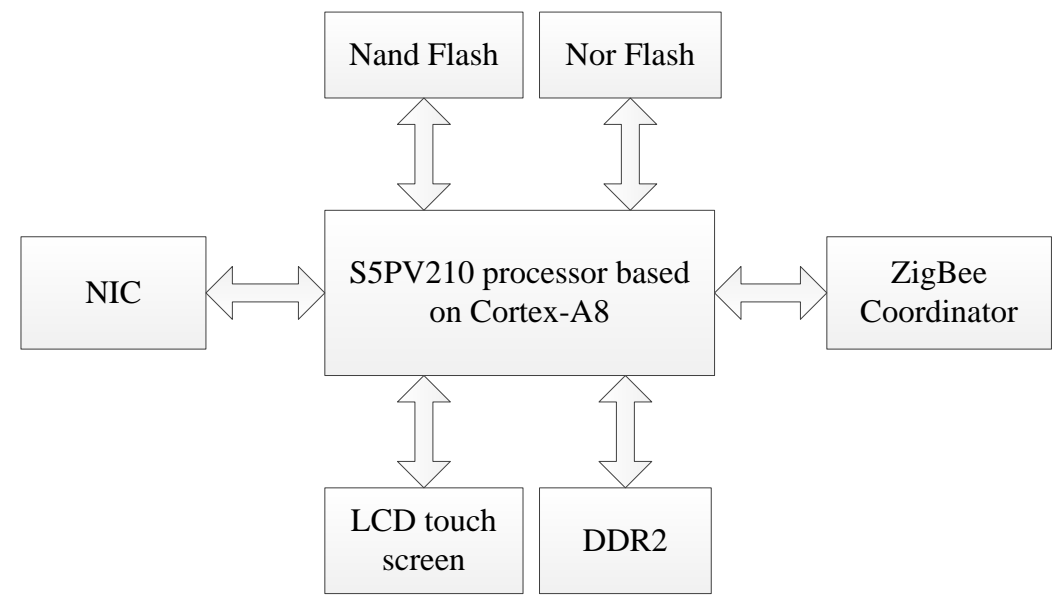

Figure 4. Hardware Structure Diagram of Embedded Gateway

\section{Software Design of System}

\subsection{Software Design of ZigBee Node}

The system uses ZigBee technology to build the star structure of wireless sensor network. The wireless sensor network mainly consists of ZigBee coordinator node, control terminal node and acquisition terminal node. The coordinator node is responsible for transmitting the information and control commands between the embedded home gateway and the ZigBee network. Firstly, the identification tag (a byte of header information) is defined for each acquisition terminal node and control terminal node. The process of data transmission as follows:

(1) The acquisition terminal node sends the sensor data to the coordinator node after adding the corresponding recognition tag to the sensor. When the coordinator receives the information from the acquisition terminal node, it transmits the information to the embedded gateway through the RS232. The embedded gateway can judge the data of the sensor by identifying the tag.

(2) When the control terminal node added to wireless sensor networks set up by the coordinator node, it will send a byte of identification tag. When the coordinator node receives the information from the control terminal node, the short address of the control terminal node is recorded, and it together with the identification tags will be send to the embedded gateway through the RS232. If the embedded gateway judges the control terminal through the identification tag, then the short address is stored, so as to send the control command through the short address. The exact program flow chart is shown in Figure 5 . 


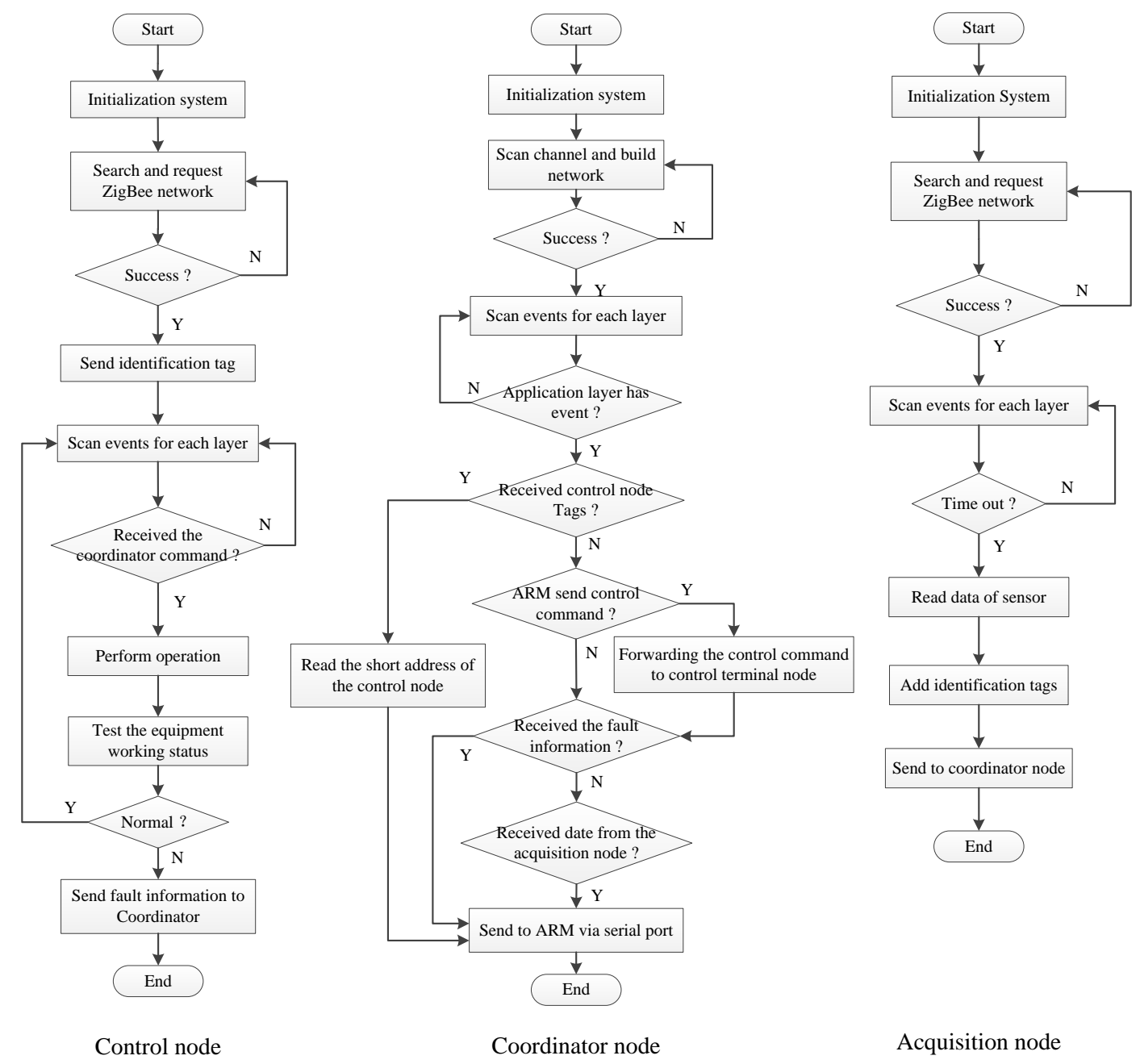

Figure 5. The Program Flow Chart of ZigBee Node

\subsection{Software Design of Embedded Gateway}

The operating system of the embedded gateway adopts the Linux operating system with the version of 2.6.38. According to the need, the standard Linux operating system is cut and configured, and then used in the ARM embedded hardware platform. We also need making the yaffs 2 root file system which the Linux needed and transplant the embedded Web server (Boa server) on the Linux operating system and write the program of man-machine operation interface by using the Qt. Qt is a cross-platform GUI system and it can achieve the powerful function of user operation interface design. The man-machine interface flow chart is shown in Figure 6. 


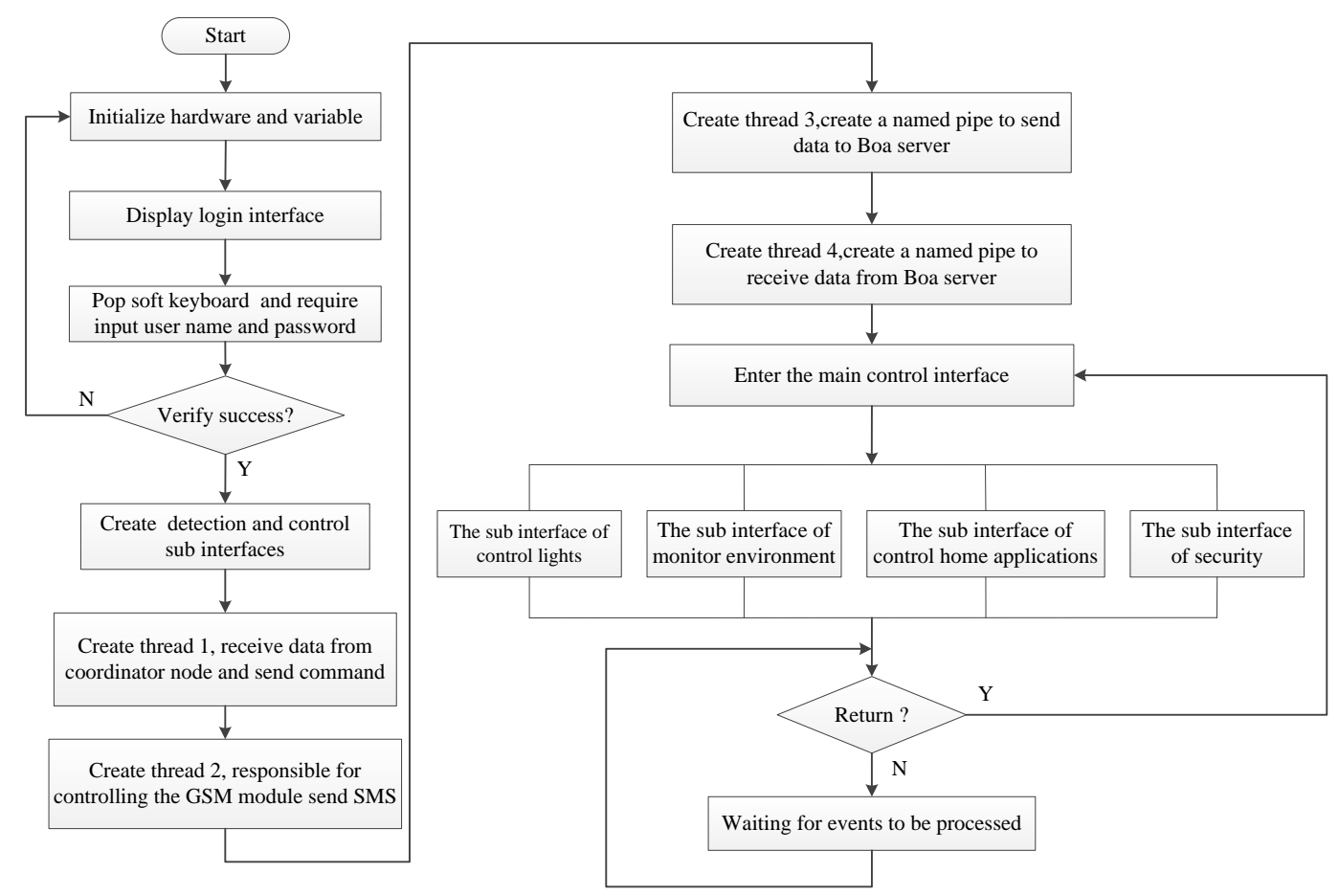

Figure 6. The Program Flow Chart of Man-machine Interface

\subsection{Design of monitoring Web}

Because of the generality of HTML language and its platform independence, so HTML language is used as a tool for web document design. However, the HTML language does not support the interaction between the browser and the server, which need to add the JavaScript to enhance the interaction between the browser and the server. Because the Boa server supports CGI script function, this provides the interface for the application of the web call background.

(1) Send control command

When the user clicks on the control button on the web page, the application will be called by the CGI script. It sends data to the Qt program by the named pipe, so as to realize the inter process communication.

(2) Receive acquisition data

The system uses AJAX asynchronous load mode to call the CGI program to request the data of Boa server. The format of data Boa server returned to the JavaScript is JSON. JavaScript analyze the data and update the results of the Web.

AJAX is a technology for creating fast and dynamic web pages. Through a small amount of data exchange with the web server, AJAX can make the web page asynchronous update. This means that the page can be updated without loading the whole page.

\section{Test and Experimental Data Analysis of the System}

\subsection{The Data Transmission Test between ZigBee Wireless Network Nodes}

After the design of the hardware and software, the experiment and debug of the system are carried out in the laboratory. In order to verify the performance of ZigBee communication in smart home system, two sets of performance indexes are tested: (1) in the absence of obstacles, with the increase of distance, test the change of packet loss rate, receiving power and spatial attenuation; (2) When the data transmission distance is $30 \mathrm{~m}$, 
the packet loss rate increases with the increase of the obstacle. Experimental data are shown in table 1 and table 2. Received power is measured in the RSSI (received signal strength) on the CC2530 chip. The value of the RSSI is stored in the register RSSIL.RSSI_VAL, the corresponding relationship between the received power is as follows:

$$
P=R S S I_{-} V A L+R S S I_{-} \text {OFFSET }[\mathrm{dBm}]
$$

RSSI_OFFSET is the experience value, about $-45 \mathrm{~dB}$.

Table 1. Test Data without Obstacle

\begin{tabular}{cccc}
\hline $\begin{array}{c}\text { Transmission } \\
\text { distance } / \mathrm{m}\end{array}$ & $\begin{array}{c}\text { Spatial attenuation } \\
/ \mathrm{dB}\end{array}$ & Packet loss rate & $\begin{array}{c}\text { Received power } \\
\text { /dBm }\end{array}$ \\
\hline 10 & 60 & $0 \%$ & -57 \\
20 & 66 & $0 \%$ & -66 \\
30 & 70 & $0 \%$ & -68 \\
40 & 72 & $0 \%$ & -69 \\
50 & 74 & $0 \%$ & -72 \\
60 & 76 & $0 \%$ & -72 \\
\hline
\end{tabular}

Table 2. Test Data of $\mathbf{3 0}$ Meters in the Presence of Obstacles

\begin{tabular}{ccccc}
\hline Obstacles & One wall & Two walls & Three walls & Four walls \\
\hline Packet loss rate & $0 \%$ & $1 \%$ & $35 \%$ & $86 \%$ \\
\hline
\end{tabular}

The experimental results show that when the transmission distance within 80 meters and there are no obstacles the packet loss rate of the transmission data is lower than $1 \%$, which can meet the needs of the normal smart home environment. When the distance between the ZigBee nodes is 30 meters and there are 2 walls the packet loss rate is only $1 \%$, if there is 1 wall the packet loss rate is $0 \%$, so it fully meet the requirements of system design.

\subsection{Overall System Test}

In the 200 square metres of laboratory evenly placed 8 terminal control nodes to control the fluorescent lamp, ceiling fans and so on; 8 acquisition terminal nodes to collect temperature and humidity, light intensity, gas, smoke and other various parameters. In order to be closer to the home indoor environment, between the 4 control terminal nodes and the 4 terminal nodes and the coordinator, the barrier is set up respectively with the separator. We connect a computer and the embedded gateway to the Internet network and turn on the power, then open turn on the power of each acquisition terminal node and control terminal node. After the start of the embedded Linux system, the Boa server and QT application are automatically launched. The operating interface of the home embedded gateway is shown in Figure 7, 8. 


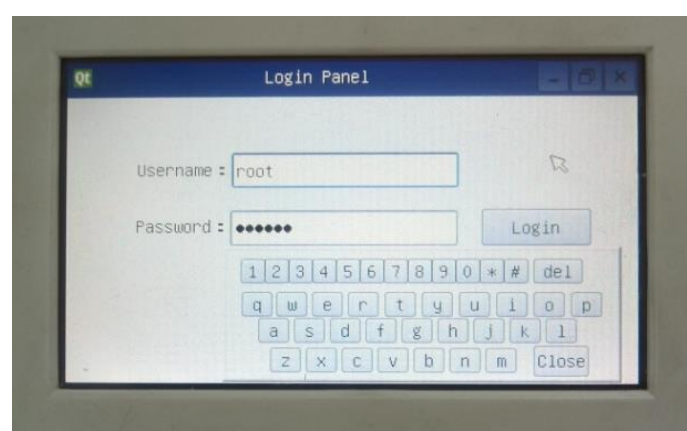

Figure 7. The Login Interface of Gateway

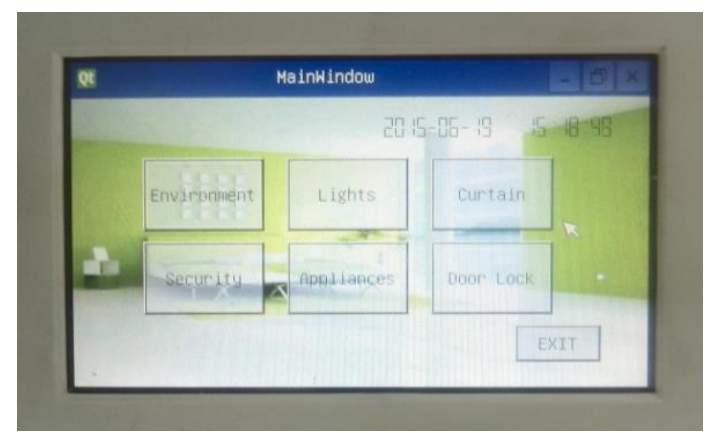

Figure 8. The Mainly Interface of the Gateway

After open the PC browser and enter the IP address of the embedded gateway in the address bar we can see the login interface. If we enter the correct user name and password, we can $\log$ in to the monitoring and control page of the smart home system to remotely monitor the home appliance equipment. As shown in Figure 9 and 10.

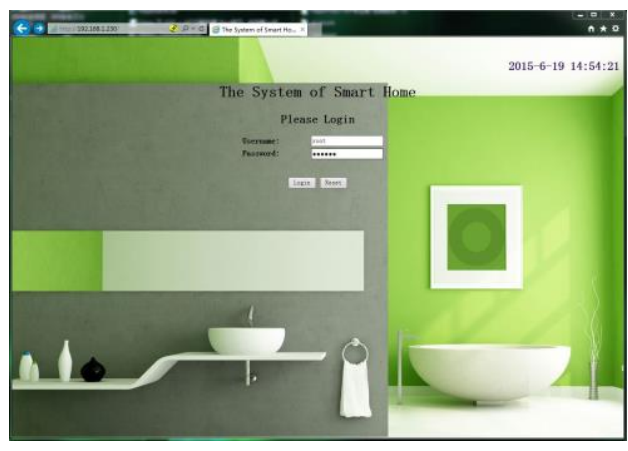

Figure 9. The Login Interface on the PC

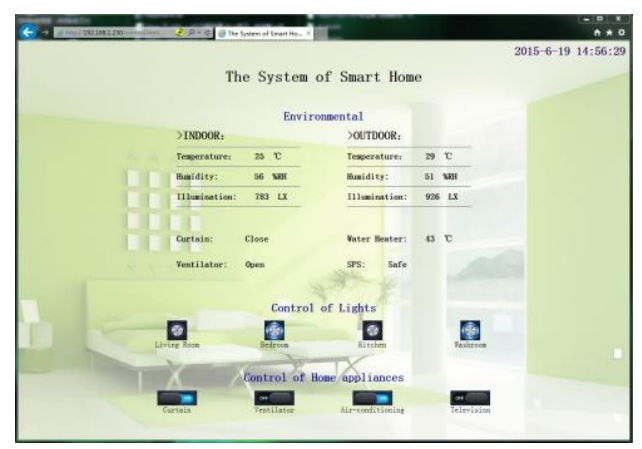

Figure 10. The Control Interface on the PC

After connect the cellphone to the network, and then turn on the browser on the phone to enter the IP address of the embedded gateway in the address bar, we can see the login interface. If we enter the correct user name and password, we can log in to the monitoring and control page of the smart home system to remotely monitor the home appliance equipment. As shown in Figure 11 and 12. 


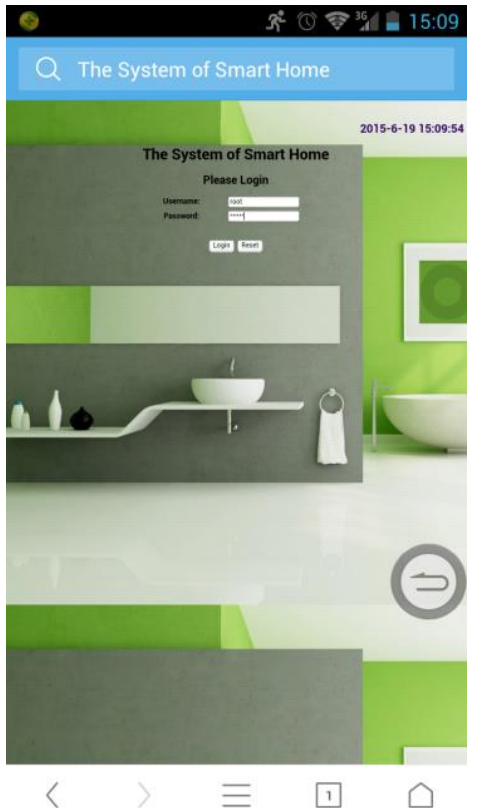

Figure 11.The Login Interface on the Cellphone

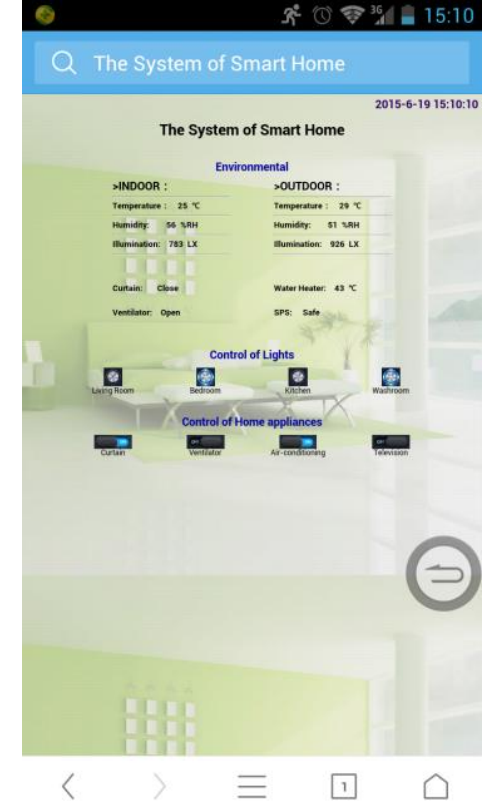

Figure 12. The Control Interface on the Cellphone

The experiment shows that the system can realize the function of the remote control of the household electrical equipment and the monitoring of the environment parameters stably.

\section{Conclusions}

The smart home system designed in this paper is based on the embedded ARM technology and ZigBee wireless sensor network technology, which can meet the purpose of the intelligent, the low cost and the portable. The test shows that the system is safe and reliable and stable. The user can not only remote control and inquire the status of the household electrical appliances but also at local can also achieve it by the LCD touch screen on the Embedded gateway. The system is easy to expand in function. For example, if there are elderly people in the home, we can add the terminal node to monitor the health of the aged at anytime and anywhere. The design of this system provides a positive reference for the development of smart home system. It can play a certain role in the broad prospects of smart home.

\section{Acknowledgements}

This project has been supported by Liaoning Provincial Aeronautical Science Foundation of China [2014ZA54006].

\section{References}

[1] C. Zhenjun, Z. Qiuxia, L. Yamin, "The research and design of smart home system based on ARM and ZigBee", Journalof China Sciencepaper, vol.10, no. 5, (2015), pp. 584-587.

[2] Z. Guangnan, "Design of smart home system based on S5PV210", Journal of Electronic Design Engineering, vol. 22, no. 8, (2014), pp. 172-174.

[3] N. Chunhun, L. Bo and W. Ying, "The design of embedded smart home system based on Web technology, J. Video Engineering", vol. 37, no. 3, (2013), pp. 86-89.

[4] Z. Xiong, Q. Huibing, H. Ming, L. Qi, M. Xiang Gen, "The design of intelligent home control system based on ZigBee", Journal, vol. 22, no. 24, (2014), pp. 180-183. 
[5] Z. Lejiang, D. Sanlei, Z. Shan, "The research on the technology of smart home based on ZigBee, J. Guangxi power", vol. 35, no. 2, (2012), pp. 17-19.

[6] Y. Jianfeng, G. Xuzhan, "The smart home system based on Zigbee Technology", Journal of Wireless Internet Technology, vol. 10, (2012), pp. 53-88.

[7] Z. Jianhua, "The portable smart home terminal system based on ARM and ZigBee", Journal of Computer Engineering and Design, vol. 29, no. 12, (2008), pp. 3064-3067.

[8] G. Hong, Z. Shijuan, "The design of embedded ZigBee gateway based on ARM S3C2410", Journal of Henan Science, vol. 30, no. 8, (2012), pp. 1072-1075.

[9] P. Yong, L. Guangming, "Research on improvement of smart home system based on ZigBee", Journal of Computer Engineering and Design, vol. 35, no. 5, (2014), pp. 1547-1550.
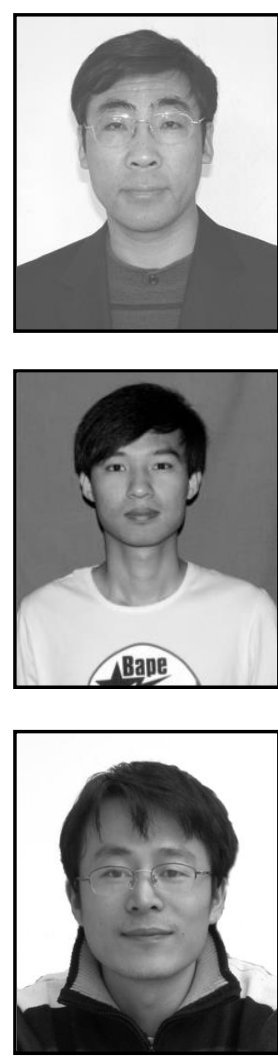

\section{Authors}

Feng Tian, He received the Ph.D. degree in detection technology and automatic equipment from Northeastern University, China, in 2004. He is currently professor at the College of Automation of Shenyang Aerospace University. He is a member of $\mathrm{CCF}$, and a doctoral tutor. His current research focuses on wireless sensor networks, internet of things, embedded systems and intelligent control.

Shimin Wang, He received the bachelor degree in automation from Dezhou University, China, in 2013.He is currently studying for a master degree in the measurement technique and automation equipment of Shenyang Aerospace University.

Yongtao Yu, He received the Ph.D. degree in control theory and control engineering from Harbin Engineering University, China, in 2013. He is currently a lecturer at the College of Automation of Shenyang Aerospace University. His current research focuses on Aircraft control and simulation. 